\title{
Effects of medical communication curriculum on perceptions of Korean medical school students
}

\author{
Hyo Hyun Yoo', Sein Shin $^{2}$ and Jun-Ki Lee ${ }^{3}$ \\ ${ }^{1}$ Department of Medical Education, Chonbuk National University School of Medicine, Jeonju, ${ }^{2}$ Department of \\ Biology Education, Chungbuk National University, Cheongju, and ${ }^{3}$ Division of Science Education, Chonbuk National \\ University College of Education, Jeonju, Korea
}

Purpose: The study examines changes in students' self-assessment of their general communication (GC) and medical communication (MC) competencies, as well as perceptions of $\mathrm{MC}$ concepts.

Methods: Participants included 108 second year medical students enrolled at a Korean medical school studying an MC curriculum. It was divided into three sections, and participants responded to questionnaires before and after completing each section. To assess perceived GC and MC competency, items based on a 7-point Likert scale were employed; a single open-ended item was used to examine students' perceptions of MC. Statistical analysis was conducted to gauge GC and MC competency, whereas semantic network analysis was used to investigate students' perceptions of MC.

Results: Students perceived their GC competency to be higher than MC. Perceived MC competency differed significantly across the three sections, whereas no differences were found for GC. There were no statistically significant differences after completing the curriculum's second and third sections; however, the vocabulary students used to describe MC concepts became more scholarly and professional. In the semantic networks, the link structure between MC-related words decreased in linearity and looseness, becoming more complex and clustered. The words 'information' and 'transfer' proved integral to students' perceptions; likewise, 'empathy' and 'communication' became closely connected in a single community from two independent communities.

Conclusion: This study differed from prior research by conducting an in-depth analysis of changes in students' perceptions of $M C$, and its findings can be used to guide curriculum development.

Key Words: Perception, Communication, Concepts, Curriculum

\section{Introduction}

Medical communication (MC) is a reciprocal process wherein doctors ask patients questions to understand their conditions, and patients respond to describe a given condition. Despite advances in medicine, medical techniques, and equipment, reciprocal communication re- mains a fundamental practice. Since MC occurs at all stages (and because its quality is directly linked to results), it is essential in medical practice. MC enhances empathy and trust between doctors and patients, which positively affects the treatment process/results, increases patient satisfaction with diagnoses, and improves job satisfaction among doctors [1-4]. Many studies indicate that communication competence is acquired and
Received: Nobemver 21, 2017 • Revised: January 23, 2018 • Accepted: August 6, 2018 Corresponding Author: Jun-Ki Lee (https://orcid.org/0000-0001-7715-5797)

Division of Science Education, Chonbuk National University College of Education, 567 Baekje-daero, Deokjin-gu, Jeonju 54896, Korea

Tel: +82.63.270.2778 Fax: +82.63.270.2781 email: junki@jbnu.ac.kr
Korean J Med Educ 2018 Dec; 30(4): 317-326.

https://doi.org/10.3946/kjme.2018.106

eISSN: 2005-7288

(C) The Korean Society of Medical Education. All rights reserved. This is an open-access article distributed under the terms of the Creative Commons Attribution Non-Commercial License (http:// creativecommons.org/licenses/by-nc/3.0/), which permits unrestricted non-commercial use, distribution, and reproduction in any medium, provided the original work is properly cited. 
improved through education [5-7], and maintained even after such training has ended [8]. Thus, institutions offering basic and continuing medical education encourage doctors to form trusting relationships with patients and develop MC skills [9-11].

Korean patients indicate that doctors are apathetic, do not listen, dismiss their concerns, offer difficult explanations, and fail to provide feedback characteristics that cause alienation [12]. Hence, Korean patients are not dissatisfied with doctors' competencies, but their communication skills. The Korean Medical Society recognizes the importance of communication, and takes steps nationally and at medical schools to improve communication competency among prospective and currently practicing doctors. Medical schools are setting learning outcomes and developing/implementing curricula with communication competency in mind. Since 2010, the Korean Medical Licensing Examination has included items from the Clinical Performance Examination regarding topics such as efficient questioning, active listening, empathy, adequate explanations, patientdoctor relations, and $\mathrm{MC}$ competency.

Efforts to improve communication competency among prospective doctors should not only encompass teaching communication skills, but nurturing appropriate and sustainable communication attitudes [13]. To effectively maintain MC competency, communication education should be initiated in a lecture-based curriculum (LBC), and continue onward to clerkship, residency, and throughout a doctor's career. Ensuring that a systematic MC curriculum occurs prior to clerkship is particularly important to fortifying the effects of $\mathrm{MC}$ education during residency [14].

Research regarding pre-clerkship MC education has focused on topics such as improving empathy [7], teaching strategies, and curriculum development [6,15]. There are numerous ways to study the outcomes of MC education; nevertheless, instructors are primarily concerned with how approaches affect students' perceptions of the subject. Constructivist views on education emphasize the importance of assessing prior knowledge and perceptions to plan curricula accordingly, as changes in perception increase the likelihood of modifications to behaviors and thoughts [16,17].

Students' perceptions of MC are expressed both verbally and nonverbally, and undoubtedly affect patient treatment. However, few studies have examined changes in students' perceptions of MC. Earlier works employed experimental research, comparative studies, literature reviews based on closed-ended surveys, and interviews [18]; few studies utilized open-ended questionnaires. However, open-ended questionnaires could be useful to understand substantive students' perception of $\mathrm{MC}$ effectively by allowing students to describe their perception more accurately and freely without fixed framework. Thus, this study attempted to understand medical students' perception of MC with written response about the meaning of MC. Moreover, considering the possibility that students' perceptions would diverse and complex, this study used semantic network analysis. Semantic network analysis assumes that text as a network by representing the words that constitute text as nodes and the co-occurrences of pair of word as links. By analyzing the topological structure of this text network, it is possible to grasp the various perceptions of individuals or groups represented in text. This approach is based on the assumption that people's perceptions or understanding toward something is constructed with links among fragmented concepts [19]. And this approach also assumed that the perception is represented in the text which is consisted of connection of words.

Since the semantic network reflects the relationships (i.e., frequency of $\mathrm{co}^{-}$occurrences) of individual words in whole text, semantic network analysis can provide a 
comprehensive understanding of the diverse and various perceptions of group about particular concept. And it is possible to macroscopically understand the major perception of the group about some concept of subject by visualizing whole network [20]. In addition, more objective study is possible with text that has been mostly dealt with subjective interpretation, because it can be quantitatively interpreted with several quantitative indexes about topological characteristics of network and each word. Semantic network analysis has been used to grasp the perception or belief about particular concepts [21,22]. Therefore, to obtain data for future MC research and promote curriculum reform, this study uses semantic network analysis to examine changes in perceptions of $\mathrm{MC}$ and perceived competency.

\section{Methods}

\section{Participants}

Participants included 108 second year medical students (65 males and 43 females) enrolled at the Chonbuk National University School of Medicine, Korea. Listwise deletion was utilized to ensure that only students who completed all surveys were included in the analyses. Thus, the analyses for perceived MC competency and perceptions of MC comprised 94 students (53 males and 41 females) and 83 students (51 males and 33 females), respectively.

\section{Curriculum}

The curriculum used in this study was based on the Analysis, Design, Development, Implementation, Evaluation model, and comprised two parts: the LBC and clinical skills-based curriculum (CBC).

Instruction in LBC focuses on general communication
(GC) and then theoretical knowledge. Students were in their second year during the first quarter, and there were 9 class hours in total spanning 4 weeks. Topics included basic communication knowledge/techniques, understanding oneself/others, MC procedures/structure, and communication in various situations. GC was added since 76 students (70.1\%) had not covered this subject prior to medical school. To ensure effectiveness, various instructional methods were used (e.g., lectures, role playing, Myers-Briggs Type Indicator workshops, demonstrations, case studies, feedback, and self-reflections). In contrast, CBC emphasizes the application of LBC theories to scenarios involving standardized patients. During the second and third quarters, groups of six to eight students practiced for 2 hours at a simulation center. These simulations on standardized patients were recorded, and tutors/classmates provided feedback for self-reflection.

\section{Study design}

Three surveys (pre-LBC, post-LBC, post-CBC) were conducted in March, June, and October 2014, respectively. The questionnaires contained two 7-point Likert scale items to assess GC and MC competence, in addition to an open-ended item regarding students' perceptions of MC. A questionnaire consisting of the following two items was presented to the participants in order to understand the perception of the medical graduate students. "Please describe what general communication is, as you know it" and "Please describe what medical communication is, as you know it." Participants were free to write their thoughts on these questions.

\section{Data analysis}

\section{1) Self-assessment of communication}

Using PASW SPSS ver. 18.0 (SPSS Inc., Chicago, IL, USA), a paired t-test was conducted between pre- and post-LBC, pre- and post-CBC, and pre- and post-CBC 
to identify differences in perceived $\mathrm{GC}$ and $\mathrm{MC}$ competency.

2) Analysis of changes in students' perceptions of $\mathrm{MC}$

Analysis of changes in students' perceptions of $\mathrm{MC}$ comprised three stages: pre-processing, network matrix construction, and analysis with visualization. NetMiner ver. 4.0 (Cyram, Seongnam, Korea) was used for semantic network analysis. The following subsections provide a detailed explanation of the aforementioned stages.

\section{3) Pre-processing}

To prepare students' responses for analysis, five pre-processing steps were performed. First, KrKwic, a Korean language analytical program, was used to segment the responses [23]. Second, words with identical morphemes but different postpositions were converted into single morphemes (e.g., 'empathized,' 'emphasizing, and 'with empathy' were converted into 'empathy'). Third, conjunctions and demonstrative pronouns such as 'or' and 'this' respectively were excluded. Fourth, only the 30 most frequently used words were selected. Fifth, the words 'patient' and 'doctor' were excluded, as both were used repeatedly in descriptions. Words that $\mathrm{co}^{-}$ occur with others have an approximate degree centrality (DC) of 1, and often become monopolizing nodes, thereby making the analysis of semantic structures difficult. Thus, 28 words were selected for semantic network analysis.

\section{4) Network construction}

Semantic networks contain information regarding relationships between words. This study assumes that words co-occurring in a response have a link connection. Word co-occurrence frequency measures how often specific words are used alongside other words, and is reflected by weight of link. Higher co-occurrence frequency directly correlates with a greater weight of link.

\section{5) Network analysis}

Semantic networks, which are based on word $\mathrm{co}^{-}$ occurrence matrices, were approached from two perspectives. First, this research confirms DC and betweenness centrality $(\mathrm{BC})$. A node's $\mathrm{DC}$ indicates how many nodes it is connected to. DC is based on the sum of link weights; thus, it reflects how strongly a node is connected to others. BC indicates how often a node functions as a broker between two other nodes. Higher degrees of $\mathrm{BC}$ suggest that a word is integral to a concept structure. Second, the network was visualized, and subcommunities comprising the network were confirmed. Only links with a weight exceeding 0.3 were visualized. In the network, a node's size correlates with DC; a line's color correlates more strongly with weight link. Subcommunities were identified according to node concentration and link weight.

\section{Ethics statement}

Informed consent was obtained from this study's participants, and the authors abided by the ethical standards outlined by Chonbuk National University's institutional review board (IRB approval no., 2016-03015-001).

\section{Results}

\section{Perceived communication competency}

Comparing students' perceived GC and MC competency showed lower levels for MC (Table 1); GC competency levels were not significantly different between $\mathrm{t}$-tests. $\mathrm{MC}$ competency improved post-LBC when compared to pre-LBC $(\mathrm{t}=-6.84, \mathrm{p}<0.01)$, and competency was higher post-CBC when compared to pre-LBC $(t=-5.83, p<0.01)$. There was no significant 
Table 1. Nonverbal Factors in a Mute State and in Speech, and Their Examples

\begin{tabular}{|c|c|c|c|c|c|c|}
\hline & \multicolumn{2}{|c|}{ Lecture-based curriculum } & \multirow{2}{*}{$\begin{array}{l}\text { Post-clinical skills-based } \\
\text { curriculum (NA) }\end{array}$} & \multicolumn{3}{|c|}{ Paired t-test } \\
\hline & Pre (PP) & Post (NC) & & $P P \& N C$ & $P P \& N A$ & $N C \& N A$ \\
\hline General communication & $4.63 \pm 1.04$ & $4.72 \pm 0.88$ & $4.59 \pm 0.86$ & $\begin{array}{c}-1.07 \\
\end{array}$ & 0.32 & 1.11 \\
\hline Medical communication & $3.51 \pm 1.09$ & $4.30 \pm 0.90$ & $4.28 \pm 0.83$ & $-6.84^{* *}$ & $-5.83^{* *}$ & 0.17 \\
\hline
\end{tabular}

Data are presented as mean \pm standard deviation or number.

PP: Pre-processing, NC: Network construction, NA: Network analysis.

${ }^{* *} \mathrm{p}<0.01$.

Table 2. DC and BC Indices for the 28 Most-Used Words

\begin{tabular}{|c|c|c|c|c|c|c|c|c|c|c|c|}
\hline \multicolumn{4}{|c|}{ Pre-LBC } & \multicolumn{4}{|c|}{ Post-LBC } & \multicolumn{4}{|c|}{ Post-clinical skills-based curriculum } \\
\hline Word & $\mathrm{F}$ & $\mathrm{DC}$ & $\mathrm{BC}$ & Word & $\mathrm{F}$ & $\mathrm{DC}$ & $\mathrm{BC}$ & Word & $\mathrm{F}$ & DC & $\mathrm{BC}$ \\
\hline Transfer & 15 & 0.134 & 0.075 & Transfer & 14 & 0.192 & 0.040 & Information & 28 & 0.195 & 0.051 \\
\hline Communication (1) & 10 & 0.119 & 0.068 & Communication (2) & 19 & 0.188 & 0.021 & Transfer & 20 & 0.179 & 0.037 \\
\hline Understanding & 16 & 0.103 & 0.062 & Conversation & 19 & 0.186 & 0.030 & Emotion & 10 & 0.167 & 0.035 \\
\hline Needs & 13 & 0.101 & 0.036 & Communication (1) & 5 & 0.183 & 0.023 & Understanding & 21 & 0.162 & 0.041 \\
\hline Treatment & 6 & 0.096 & 0.028 & Relationship & 9 & 0.170 & 0.024 & Process & 16 & 0.160 & 0.027 \\
\hline Communication (2) & 10 & 0.094 & 0.023 & Empathy & 16 & 0.161 & 0.028 & Empathy & 14 & 0.141 & 0.031 \\
\hline Conversation & 10 & 0.092 & 0.096 & Formation & 9 & 0.147 & 0.006 & Communication (1) & 6 & 0.140 & 0.017 \\
\hline Knowledge & 9 & 0.092 & 0.032 & Trust & 4 & 0.147 & 0.008 & Formation & 7 & 0.122 & 0.017 \\
\hline Situation & 9 & 0.091 & 0.029 & Information & 23 & 0.145 & 0.023 & Accuracy & 8 & 0.121 & 0.011 \\
\hline Explanation & 10 & 0.089 & 0.040 & Treatment & 12 & 0.143 & 0.026 & Communication (2) & 12 & 0.119 & 0.014 \\
\hline Talk & 5 & 0.088 & 0.039 & Accuracy & 6 & 0.137 & 0.011 & Information transfer & 4 & 0.116 & 0.002 \\
\hline Accuracy & 9 & 0.085 & 0.015 & Understanding & 14 & 0.130 & 0.022 & Needs & 6 & 0.115 & 0.006 \\
\hline Information & 12 & 0.083 & 0.039 & Professional & 7 & 0.125 & 0.007 & Condition & 8 & 0.114 & 0.010 \\
\hline Ability & 7 & 0.076 & 0.014 & Needs & 23 & 0.123 & 0.015 & Between & 7 & 0.113 & 0.015 \\
\hline Process & 3 & 0.075 & 0.007 & Situation & 7 & 0.121 & 0.007 & Situation & 11 & 0.108 & 0.018 \\
\hline Grasp & 4 & 0.063 & 0.007 & Care & 6 & 0.118 & 0.009 & Thought & 4 & 0.107 & 0.009 \\
\hline Aim & 5 & 0.060 & 0.010 & Knowledge & 4 & 0.117 & 0.003 & Relationship & 4 & 0.105 & 0.011 \\
\hline Active listening & 5 & 0.059 & 0.008 & Diagnosis & 8 & 0.113 & 0.009 & Result & 4 & 0.105 & 0.013 \\
\hline Person & 7 & 0.057 & 0.003 & Specific & 5 & 0.112 & 0.002 & Sharing & 5 & 0.104 & 0.017 \\
\hline Saying & 3 & 0.057 & 0.013 & Active listening & 6 & 0.104 & 0.012 & Explanation & 6 & 0.095 & 0.008 \\
\hline Listening & 3 & 0.055 & 0.002 & Saying & 10 & 0.102 & 0.010 & Language & 4 & 0.095 & 0.002 \\
\hline Oneself & 5 & 0.054 & 0.005 & Position & 9 & 0.096 & 0.007 & Knowledge & 7 & 0.091 & 0.006 \\
\hline Professional & 5 & 0.054 & 0.002 & Rapport & 6 & 0.096 & 0.001 & Opinion & 5 & 0.089 & 0.013 \\
\hline Hospital & 3 & 0.044 & 0.006 & Help & 6 & 0.086 & 0.006 & Health professional & 4 & 0.080 & 0.003 \\
\hline Empathy & 4 & 0.041 & 0.002 & Respect & 7 & 0.080 & 0.007 & Rapport & 4 & 0.075 & 0.002 \\
\hline Problem & 5 & 0.036 & 0.007 & Provision & 6 & 0.076 & 0.009 & Treatment & 5 & 0.067 & 0.002 \\
\hline Clearness & 3 & 0.036 & 0.001 & Expression & 4 & 0.066 & 0.005 & Position & 6 & 0.062 & 0.006 \\
\hline Protector & 3 & 0.034 & 0.001 & Talk & 9 & 0.064 & 0.002 & Conversation & 10 & 0.053 & 0.004 \\
\hline
\end{tabular}

DC: Degree centrality, BC: Betweenness centrality, LBC: Lecture-based curriculum.

difference in perceived MC competency post-LBC or post-CBC.

\section{Students' perceptions of medical com- munication}

The 30 most frequently used words were verified
(Table 2). 'Patient' was used most frequently (65, 104, and 96 times), and 'doctor' second most frequently (29, 53, and 46 times). The third most frequently used words were 'understanding' pre-LBC, 'knowledge' and 'needs' post-LBC, and 'knowledge' post-CBC.

In examining DC among the words (excluding 'patient' 
and 'doctor'), the three highest levels of pre-LBC DC were $0.134,0.119$, and 0.103 for 'transfer,' 'communication,' and 'understanding,' respectively; $\mathrm{BC}$ levels were also the highest for these words $(0.075,0.068$, and 0.062, respectively). Post-LBC, DC levels were highest for 'transfer,' 'communication,' and 'conversation' (0.192, 0.188 , and 0.186, respectively); $\mathrm{BC}$ was highest for 'transfer' (0.040), followed by 'conversation' (0.030) and 'empathy' (0.028). Thus, 'transfer' and 'conversation' played integral roles in the network. Post-CBC, DC was

Fig. 1. Pre-Lecture-Based Curriculum Network

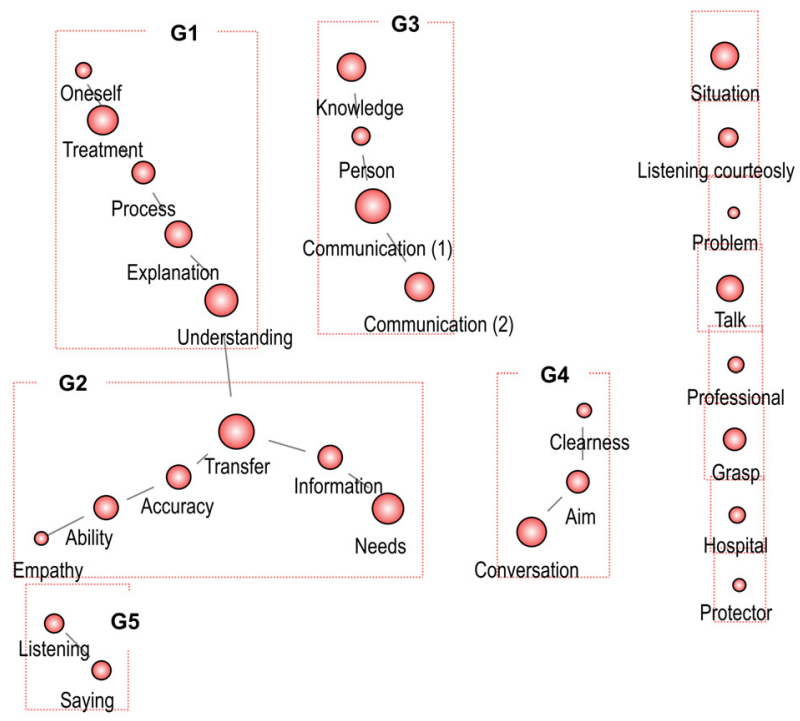

Fig. 2. Post-Lecture-Based Curriculum Network

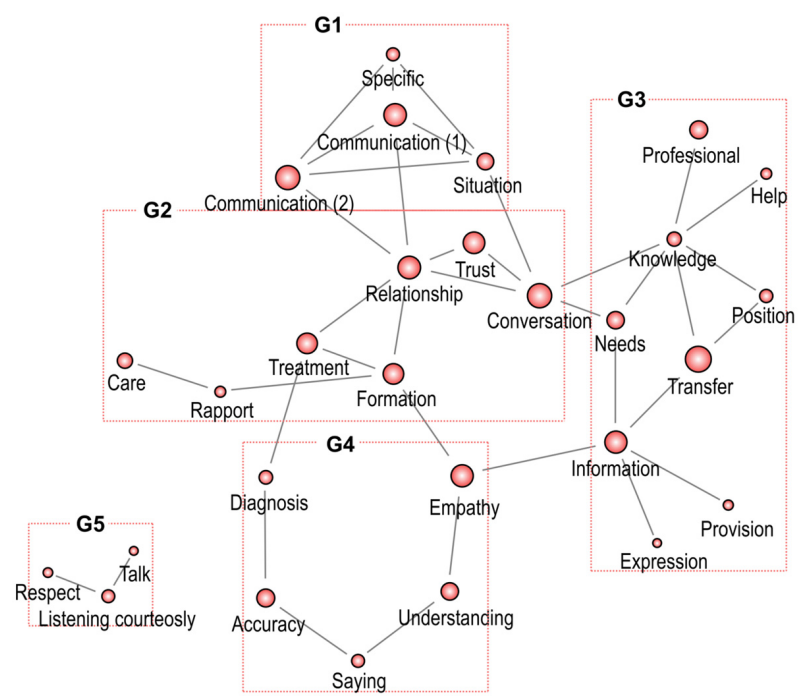

highest for 'information' (0.195), followed by 'transfer' (0.179) and 'emotion' (0.167); 'information' also had the highest $\mathrm{BC}$ (0.051), followed by 'understanding' (0.041) and 'transfer' (0.037). Thus, 'information' was an integral part of the network post-CBC. In addition, DC for 'information' increased for each stage (from 0.083, to 0.145 , and then 0.195).

To understand the network's structure, subcommunities were identified and networks visualized. Nodes with $\mathrm{BC}$ values exceeding 0.3 were visualized to focus on their most integral structures. The visualized pre-LBC, post-LBC, and post-CBC network densities were 0.042, 0.099, and 0.091, respectively. Network density doubled post-CBC. Although each period had five sub-communities, their structural features differed. As Fig. 1 shows, pre-LBC had five sub-communities: 'explanation' and 'treatment' (G1), 'information' and 'transfer' (G2), 'communication' (G3), 'conversation' (G4), and 'listening' and 'saying' (G5). Eight other words appeared in an isolated node with weak ties; virtually no connections existed between sub-communities, and each had linear, generally loose structures.

The five post-LBC sub-communities were 'commu-

Fig. 3. Post-Clinical Skills-Based Curriculum Network

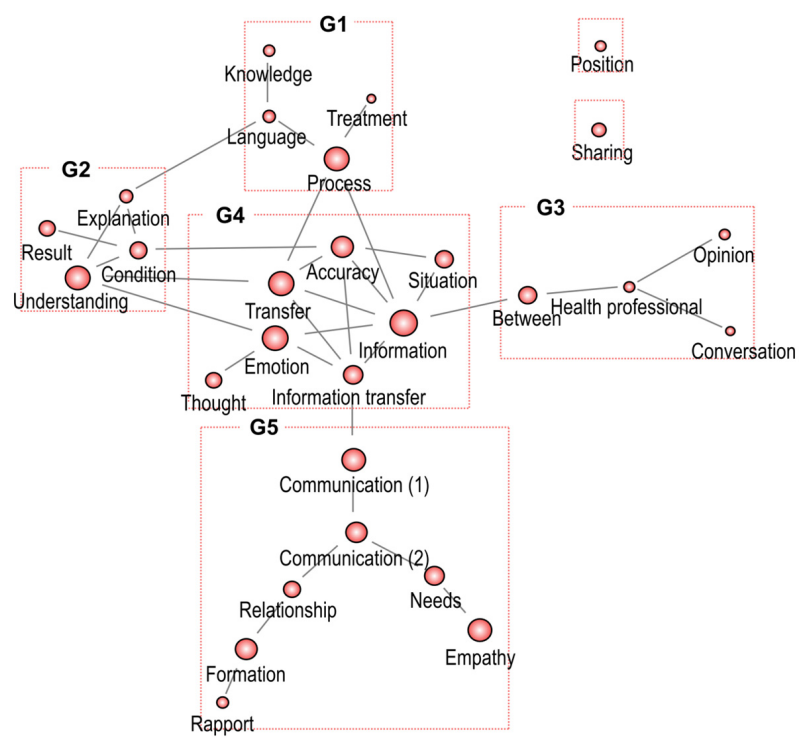


nication' (G1), 'empathy' and 'understanding' (G2), 'relationship' and 'formation' (G3), 'information' and 'transfer' (G4), and 'listening courteously' (G5) (Fig. 2). When compared to pre-LBC, the network had far more connections between and within sub-communities, as well as increased structural complexity. The post-CBC network had two isolated nodes and five sub- communities: 'empathy' and 'communication' (G1), 'condition' and 'explanation' (G2), 'information transfer' (G3), 'health professional' and 'conversation' (G4), and 'process' and 'communication' (G5) (Fig. 3). G4 was a nucleus for these sub-communities.

\section{Discussion}

Students perceived themselves as having greater competency in GC. This indicates that students believed MC differs from $\mathrm{GC}$, and recognized that $\mathrm{MC}$ poses a challenge. Recent graduates from medical school experience difficulty in communicating effectively with patients [24,25]; thus, MC curricula are essential to building MC competency, which should promote effective communication with patients, guardians, and colleagues.

Perceptions of GC competency did not change, although perceived $\mathrm{MC}$ competency improved significantly post-LBC. No statistically significant increases in MC competency occurred pre- ${ }^{-}$or post-CBC; in fact, $\mathrm{MC}$ competency decreased post-CBC. This could be because students experienced difficulty in applying MC theories during the simulations. To lessen the gap between theory and practice, instructors must provide active feedback in $\mathrm{CBC}$ to induce long-term effects [26]. Also, the relationship between LBC and CBC should be strengthened and continuously monitored to improve the curriculum.

In describing $\mathrm{MC}$, students' descriptions became more scholarly and professional. For example, pre-LBC words such as 'talk,' 'listening,' and 'conversation' were replaced with 'active listening' and 'rapport' post-LBC. This demonstrates that learning about $\mathrm{MC}$ diversified participants' vocabularies, but not that concrete changes occurred regarding their perceptions of it. Therefore, potential relationships between words were explored by examining pre-LBC, post-LBC, and post-CBC semantic networks. This investigation yielded four major findings. First, connections in network structures became less linear/loose and more clustered. Prior to commencing the curriculum, students had only indirect experience with MC; as such, conceptualizing it may have been difficult. Nevertheless, as students progressed through the curriculum connections between words gradually increased.

Second, the word 'information' played a critical role in students' perceptions. It should be noted that 'information' and 'treatment' were connected to a single sub-community pre-LBC and to 'empathy' post-LBC. Post-CBC, 'information' was a hub connected to other communities (e.g., subject, technique, content, and situational context). Interestingly, issues concerning health professionals surfaced post-CBC, whereas pre-LBC patients were the primary focus. This is a result of step-by-step education, and one can conclude that $\mathrm{MC}$ perceptions evolved from being general to complex in nature.

Third, 'transfer' and 'information' played crucial roles in the semantic networks. 'Transfer' is generally used to describe a one-way transmission of information to relevant parties; however, $\mathrm{MC}$ requires information exchanges, as doctors cannot transmit information unless patients provide input. Thus, students perceived MC as being unidirectional rather than bidirectional. Information exchanges between doctors and patients lead to more accurate diagnoses, and enable doctors to under- 
stand patients' sociopsychological issues in addition to their physical pain [27]. Since the late-20th century, the patient-doctor communication model has been patient rather than disease centric [28]. Thus, doctors must actively seek patient feedback to provide effective treatment, and such efforts should begin during medical school. This can be accomplished by gauging students' experiences and adjusting school curricula accordingly. Fourth, 'empathy' and 'communication' were independent communities that became closely connected. The words had no connections pre-LBC; post-LBC they existed in independent communities, but were indirectly connected; post-CBC they joined a single community. This indicates that despite learning about empathy and communication in $\mathrm{LBC}$, students were unable to connect both concepts until they merged in $\mathrm{CBC}$. Important words like 'active listening' and 'respect' emerged post-LBC; however, with the exception of 'empathy' and 'communication' these words decreased in number post$\mathrm{CBC}$, thereby indicating that concepts such as active listening and respect were not instilled in students. Despite being core competencies, empathy and active listening were not acquired by students, which suggests that the curriculum requires refinement.

This study investigated changes in perception of MC at a Korean medical school, and was therefore conducted in Korean rather than English. Languages feature different harmonies of words and influence the interpretation of concepts. Moreover, language is closely tied to sociocultural context, and the concept of language and its usage varies between cultures. Hence, the research is limited in some respects, and this should be considered in $\mathrm{ex}^{-}$ amining its results.

Despite these limitations, the study's contributions are twofold. First, earlier research regarding MC only included results from before and/or after an intervention, and rarely provided an in-depth analysis of students' perceptions. Since this study thoroughly examined changes in students' perceptions, its results should prove beneficial in improving MC curricula. Second, the use of semantic network analysis in medical education research is relatively uncommon. Textual semantic network analysis facilitates an improved understanding of structural context, and also presents findings in the form of a quantitative index. In sum, this study contributes to specialized research regarding medical school curricula, and used multiple approaches to explore their related phenomena.

\section{ORCID:}

Hyo Hyun Yoo: https://orcid.org/0000-0003-4226-2589; Sein Shin: https://orcid.org/0000-0002-8333-7375; Jun-Ki Lee: https://orcid.org/0000-0001-7715-5797 Acknowledgements: None.

Funding: None.

Conflicts of interest: No potential conflict of interest relevant to this article was reported.

Author contributions: Design of the work, data collection, drafting the article: HHY; data anaysis and interpretation, drafting the article: SS; and data analysis and interpretation, drafting the article: JKL.

\section{References}

1. Zolnierek KB, Dimatteo MR. Physician communication and patient adherence to treatment: a meta-analysis. Med Care. 2009;47(8):826-834.

2. Ong LM, de Haes JC, Hoos AM, Lammes FB. Doctorpatient communication: a review of the literature. Soc Sci Med. 1995;40(7):903-918.

3. Rider EA, Hinrichs MM, Lown BA. A model for communication skills assessment across the undergraduate 
curriculum. Med Teach. 2006;28(5):el27-el34.

4. Maguire P, Pitceathly C. Key communication skills and how to acquire them. BMJ. 2002;325(7366):697-700.

5. Makoul G, Schofield T. Communication teaching and assessment in medical education: an international consensus statement. Netherlands Institute of Primary Health Care. Patient Educ Couns. 1999;37(2):191-195.

6. Yedidia MJ, Gillespie CC, Kachur E, et al. Effect of communications training on medical student performance. JAMA. 2003;290(9):1157-1165.

7. Winefield HR, Chur-Hansen A. Evaluating the outcome of communication skill teaching for entry-level medical students: does knowledge of empathy increase? Med Educ. 2000;34(2):90-94.

8. Fallowfield L, Jenkins V, Farewell V, Solis-Trapala I. Enduring impact of communication skills training: results of a 12-month follow-up. Br J Cancer. 2003;89(8):14451449 .

9. Association of American Medical Colleges. Contemporary issues in medicine: communication in medicine Washington, USA: Association of American Medical Colleges; 1999.

10. Accreditation Council for Graduate Medical Education. ACGME outcome project. http://www.acgme.org/outcome. Published 2009. Accessed December 3, 2015.

11. Frank JR, Jabbour M, Tugwell P, Boyd D, Labrosse J, MacFadyen J. Skills for the new millennium: report of the societal needs working group, CanMEDS 2000 Project. Ann R Coll Physicians Surg Can. 1996;29(4): 206-216.

12. Bak YI, Park EW. Problems of the curriculum for the improvement of the medical communication in Korean medical schools. Korean J Health Commun. 2009;4(4): 34-42.

13. Park EW. Development of medical communication subject in medical school curriculum. Korean J Health Commun. 2009;4(4):23-33.
14. Kurtz S, Silverman J, Draper J. Specific issues of communication curriculum design at different levels of medical education. In: Kurtz S, Silverman J, Draper J, eds. Teaching and Learning Communication Skills in Medicine. 2nd ed. Oxford, UK: Radcliffe Publishing; 2005:233-251.

15. Yoo HH, Kim JS. The development and effects of a medical communication skills program for medical school students. Korean J Med Educ. 2014;26(3):189-195.

16. Hammer D. Misconceptions or p-prims: how may alternative perspectives of cognitive structure influence instructional perceptions and intentions. J Learn Sci. 1996;5(2):97-127.

17. Limón M. On the cognitive conflict as an instructional strategy for conceptual change: a critical appraisal. Learn Instr. 2001; 11 (4-5):357-380.

18. Han HH, Kim S. Trends of communication skills education in medical schools. Korean J Med Educ. 2009; $21(1): 35-41$

19. DiSessa AA. A bird's-eye view of the 'pieces' vs. 'coherence' controversy. In: Vosniadou S, ed. International Handbook of Research on Conceptual Change. 2nd ed. New York, USA: Routledge; 2008:35-60.

20. Gloor P, Diesner J. Semantic social networks. In: Alhajj R, Rokne J, eds. Encyclopedia of Social Network Analysis and Mining. New York, USA: Springer; 2014:16541659.

21. Peters-Burton E, Baynard LR. Network analysis of beliefs about the scientific enterprise: a comparison of scientists, middle school science teachers and eighth-grade science students. Int J Sci Educ. 2013;35(16):2801-2837.

22. Kang GJ, Ewing-Nelson SR, Mackey L, et al. Semantic network analysis of vaccine sentiment in online social media. Vaccine. 2017;35(29):3621-3638.

23. Park HW, Leydesdorff L. Understanding the KrKwic: a computer program for the analysis of Korean text. J Korean Data Anal Soc. 2004;6(5):1377-1387. 
24. Klass D. The bedrock of clinical encounters. CPSO Memb Dialogue. 2001;(Sep/Oct):1 1-12.

25. Jung JW, Lee YM, Kim BS, Ahn D. Analysis of the perceived effectiveness and learning experience of medical communication skills training in interns. Korean J Med Educ. 2010;22(1):33-45.

26. Grant VJ, Hawken SJ. What do they think of it now?: medical graduates' views of earlier training in communi- cation skills. Med Teach. 2000;22(3):260-264.

27. Haq C, Steele DJ, Marchand L, Seibert C, Brody D. Integrating the art and science of medical practice: innovations in teaching medical communication skills. Fam Med. 2004;36 Suppl:S43-S50.

28. McWhinney I. The need for a transformed clinical method. In: Stewart M, Roter D, eds. Communicating with Medical Patients. London, UK: Sage; 1989:25-40. 